\title{
TAUTOLOGICAL FORMAL FROBENIUS MANIFOLD STRUCTURES ON A FROBENIUS ALGEBRA
}

\author{
JIAN ZHOU
}

\begin{abstract}
We give some tautological constructions of formal Frobenius manifold structures on a Frobenius algebra. As a consequence, one can construct a version of "quantum cohomology" for any connected oriented closed manifold, in the sense that it is a deformation given by a function which satisfies the WDVV equations. We compute the potential functions for complex projective spaces.
\end{abstract}

\section{Introduction}

A consequence of the results in this paper is that one can construct a version of "quantum cohomology" for every connected oriented closed manifold. It is well-known that the de Rham cohomology of a smooth manifold has a natural ring structure given by the wedge product. The notion of quantum cohomology is concerned with deformations of de Rham cohomology ring (algebra). The general theory of deformations of rings and algebras was developed by Gerstenhaber [11] in the sixties. However, the constructions for deformations of de Rham cohomology ring appeared much later in the nineties, with motivations from string theory. String theorists suggested a construction called quantum cohomology by Gromov-Witten invariants in symplectic geometry and enumerative algebraic geometry. Since important geometric information is encoded in such deformations, they have attracted a lot of attention.

Closely related to the study of quantum cohomology is the notion of a Frobenius manifold introduced and extensively studied by Dubrovin (see [10] and the references therein). Frobenius manifolds are used to give coordinate free formulation of WDVV (Witten-Dijkgraaf-Verlinde-Verlinde) equations (cf. [9] and [21]). We are interested in families of deformations of a Frobenius algebra which are given by potential functions. The associativity for all of the perturbed multiplications gives the WDVV equations. In Manin [17], formal power series solutions to WDVV equations are called formal Frobenius manifold structures. They give formal deformations with some nice properties.

Return now to the problem of deforming the de Rham cohomology. We are interested in finding algebraic constructions. In joint work with Cao $[2,3]$, a deformation of the wedge product on any Poisson manifold and the so-called quantum de Rham cohomology were introduced. Motivated by the idea of mirror symmetry, we also used the method of DGBV algebras $[1,17]$ to construct formal

Received May 11, 1999. 
Frobenius manifold structures on Dolbeault [4] and de Rham cohomology [5] of closed Kähler manifolds. (See also Merkulov [18].) In [5], under the natural identification of the Dolbeault cohomology with the de Rham cohomology, these formal Frobenius manifold structures are shown to be identical. The above results have been generalized to hyperkähler manifolds [6] and the equivariant cohomology [7] of Kähler manifolds.

All of the above constructions use some special geometric features of the symplectic or Kähler manifolds, hence will not apply for arbitrary manifolds. In a recent paper [22], the author used the formulas in Merkulov [19] to show that one can obtain an $A_{\infty}$ algebra structure on the de Rham cohomology of any closed oriented Riemannian manifold. In fact, there is a construction of a structure of $A_{\infty}$-algebra on the de Rham cohomology implicitly in Chen's work [8]. See e.g., Gugenheim-Stasheff [13] and Zhou [23]. Such results motivate this work. Recall that a formal Frobenius manifold structure is equivalent to a cyclic $\mathrm{Comm}_{\infty}$-algebra structure ([16], see also $\left.\S 2\right)$. In this paper, we show that for any Frobenius algebra, there is a tautological construction of a formal Frobenius manifold structure on it. Furthermore, for any Frobenius algebra with identity which satisfies some simple conditions, there is a tautological construction of a formal Frobenius manifold structure with identity on it. We also show that over $\mathbb{R}$ and $\mathbb{C}$, the potential series always converges, hence we obtain families of Frobenius algebras. In particular, when applied to the de Rham cohomology of any connected closed oriented manifold, our constructions yields what we call the tautological Frobenius structure on de Rham cohomology. As first proved by Ruan-Tian [20], quantum cohomology theory via Gromov-Witten invariants provides constructions of formal power series solutions to the WDVV equations on the de Rham cohomology of certain symplectic manifolds. So as mentioned in the abstract and the first paragraph, our construction gives a version of "quantum cohomology" for an arbitrary closed oriented manifold.

The rest of the paper is arranged as follows. In $\S 2$, we review the definition of formal Frobenius manifold structure and its equivalence with cyclic $\mathrm{Comm}_{\infty}{ }^{-}$ algebra structure. We give our tautological constructions in $\S 3$. Applications to the de Rham cohomology and calculations for $\mathbb{C P}_{n}$ appear in $\S 4$.

Acknowledgment. The work in this paper is partially supported by an NSF group infrastructure grant. The author thanks the Mathematics Department and the Geometry-Analysis-Topology group of Texas A\&M University for hospitality and financial support. Special thanks are due to Huai-Dong Cao for constant encouragement.

\section{Formal Frobenius manifold structures and Comm $_{\infty}$-algebras}

Let $\mathbf{k}$ be a commutative $\mathbb{Q}$-algebra, $H$ a free $\mathbf{k}$-module of rank $n$. Assume that $H$ is endowed with a symmetric bilinear form $g: H \otimes H \rightarrow \mathbf{k}$ which is nondegenerate in the sense that it induces an isomorphism $H \rightarrow H^{t}$, where 
$H^{t}$ denotes the dual module. Given a commutative associative multiplication . : $H \otimes_{\mathbf{k}} H \rightarrow H$, if

$$
g(X \cdot Y, Z)=g(X, Y \cdot Z)
$$

for any $X, Y, Z \in H$, then we say $g$ is an invariant inner product on the k-algebra $(H, \cdot)$, and the triple $(H, \cdot, g)$ is called a Frobenius algebra. When $1 \in H$ is an identity for the multiplication, set $\int: H \rightarrow \mathbf{k}$ by $\int X=g(1, X)$. It is called the integral on $(H, \cdot, g)$. Clearly $\int$ and $g$ determine each other:

$$
g(X, Y)=g(1, X \cdot Y)=\int X \cdot Y .
$$

Given a Frobenius algebra $(H, \cdot, g)$, its structure is determined by some constants $g_{a b}$ and $\phi_{a b}^{c}$ defined as follows. Choose a basis $\left\{e_{a}\right\}$ of $H$, then

$$
g_{a b}=g\left(e_{a}, e_{b}\right), \quad e_{a} \cdot e_{b}=\phi_{a b}^{c} e_{c} .
$$

Let $\phi_{a b c}=\phi_{a b}^{p} g_{p c}$. Then $\phi_{a b c}=g\left(e_{a} \cdot e_{b}, e_{c}\right)$. From (1), one sees that $\phi$ is symmetric in the three indices. Hence $\phi$ can be regarded as a cubic polynomial on $H$. The associativity of the multiplication is equivalent to the following system of equations

$$
\phi_{a b p} g^{p q} \phi_{q c d}=\phi_{b c p} g^{p q} \phi_{a q d}
$$

where $\left(g^{a b}\right)$ is the inverse matrix of $\left(g_{a b}\right)$. When $(H, \cdot)$ has an identity 1 , one can recover the inner product and multiplication from $\phi$ by

$$
g_{a b}=\phi_{0 a b}, \quad \phi_{a b}^{c}=\phi_{a b p} g^{p c} .
$$

Here we have taken $e_{0}=1$.

Definition 2.1. A formal Frobenius manifold structure on $(H, g)$ is a formal power series $\Phi$ (called the potential function) which satisfies the WDVV equations:

$$
\frac{\partial^{3} \Phi}{\partial x^{a} \partial x^{b} \partial x^{p}} g^{p q} \frac{\partial^{3} \Phi}{\partial x^{q} \partial x^{c} \partial x^{d}}=\frac{\partial^{3} \Phi}{\partial x^{b} \partial x^{c} \partial x^{p}} g^{p q} \frac{\partial^{3} \Phi}{\partial x^{q} \partial x^{a} \partial x^{d}},
$$

where $\left\{x^{a}\right\}$ are the linear coordinates in $\left\{e_{a}\right\}$.

Given a potential function $\Phi$, let $\phi$ denote its degree three part. Set $\phi_{a b}^{c}=$ $\phi_{a b p} g^{p c}$, then the rule

$$
e_{a} \cdot e_{b}=\phi_{a b}^{c} e_{c}
$$

defines a structure of a Frobenius algebra on $(H, g)$. If we have $\phi_{0 a b}=g_{a b}$, then $e_{0}$ is the identity for $(H, \cdot)$. The formal Frobenius manifold structure defines a formal deformation of this Frobenius algebra (with $n$ parameters) in the space of Frobenius algebras. Let $K$ be the space of formal power series on $H^{t}$, and set

$$
e_{a} \circ e_{b}=\Phi_{a b}^{c} e_{c},
$$

where $\Phi_{a b}^{c}=\frac{\partial^{3} \Phi}{\partial x^{a} \partial x^{b} \partial x^{p}} g^{p c}$. Then it is easy to see that

$$
g(X \circ Y, Z)=g(X, Y \circ Z)
$$


for any $X, Y, Z \in H_{K}=H \otimes_{\mathbf{k}} K$. Furthermore, WDVV equations imply that $\circ$ defines an associative multiplication on $H_{K}$. In fact, WDVV equations were discovered by considering deformations of Frobenius algebras, see e.g., DijkgraafVerlinde-Verlinde [9]. We will refer to the Frobenius algebra $(H, \cdot, g)$ determined by the cubic term of $\Phi$ the initial data of the WDVV equations. Assume that $(H, \cdot)$ has an identity $1=e_{0}$, a formal Frobenius manifold structure is said to have an identity if

$$
\frac{\partial^{3} \Phi}{\partial x^{a} \partial x^{b} \partial x^{0}}=g_{a b}
$$

Equivalently, $x^{0}$ does not appear in terms of degrees $\geq 4$ in $\Phi$. Since

$$
1 \circ e_{a}=\Phi_{0 b}^{c} e_{c}=\frac{\partial^{3} \Phi}{\partial x^{0} \partial x^{b} \partial x^{p}} g^{p c} e_{c},
$$

$1 \circ e_{a}=e_{a}$ for all $a$ (or equivalently, 1 is an identity for $\circ$ ) if and only if

$$
\frac{\partial^{3} \Phi}{\partial x^{0} \partial x^{b} \partial x^{p}} g^{p c}=\delta_{b c}
$$

This is equivalent to (5).

It is not always practical to directly check that a given formal power series satisfies the WDVV equations. We will use an equivalent description.

Definition 2.2. A Comm $_{\infty}$-algebra structure on a free $\mathbf{k}$-module is a sequence of symmetric products $\circ_{n}: \otimes^{n} H \rightarrow H$ which satisfies the higher associativity equations

$$
\sum_{S_{1} \amalg S_{2}}\left(\left(a, b, x_{S_{1}}\right), c, x_{S_{2}}\right)=\sum_{S_{1} \amalg S_{2}}\left(a,\left(b, c, x_{S_{1}}\right), x_{S_{2}}\right) .
$$

Here, if $S=\left\{s_{1}, \ldots, s_{k}\right\}$ is a finite set, $x_{S}$ is an abbreviation for $x_{s_{1}}, \ldots, x_{s_{k}}$, and we write $\circ_{n}\left(x_{1} \otimes \cdots \otimes x_{n}\right)=\left(x_{1}, \ldots, x_{n}\right)$. Here the sum is taken over disjoint unions $S_{1} \coprod S_{2}$ such that $S_{1} \cup S_{2}=\{1, \ldots, n\}$.

Suppose that $H$ has a nondegenerate symmetric bilinear form $g: H \otimes H \rightarrow \mathbf{k}$. Then a $\mathrm{Comm}_{\infty}$-algebra structure on $H$ is called cyclic if

$$
Y_{n+1}\left(x_{1}, \ldots, x_{n+1}\right)=Y_{n+1}\left(x_{n+1}, x_{1}, \ldots, x_{n}\right),
$$

where $Y_{n+1}\left(x_{1}, \ldots, x_{n+1}\right)=g\left(\left(x_{1}, \ldots, x_{n}\right), x_{n+1}\right)$.

In Getzler [12], Comm $_{\infty}$-algebras were called hypercommutative algebras. They also find their roots in string theory, see e.g., Dijkgraaf-Verlinde-Verlinde [9]. As shown by Manin ([16], Chapter III, Theorem 1.5), there is a one-toone correspondence between the set of formal Frobenius manifold structures on $(H, g)$ with the set of cyclic Comm $_{\infty}$-algebra structures. In fact, modulo terms of degree $\leq 2$,

$$
\Phi=\sum_{n \geq 3} \frac{1}{n !} Y_{n}
$$


Of course it is natural to extend it by

$$
Y_{2}(x, x)=g(x, x) .
$$

And when $H$ has a unit 1 , extend it further by

$$
Y_{1}(x)=g(1, x)=\int x .
$$

We will call

$$
\widetilde{\Phi}=\sum_{n \geq 1} \frac{1}{n !} Y_{n}
$$

the normalized potential function. Using the higher multiplications, the product o defined in (4) is equivalent to a multiplication given in Getzler [12]:

Lemma 2.1. For a cyclic $\mathrm{Comm}_{\infty}$-algebra structure on $(H, g)$, we have for $X, Y \in H_{K}$,

$$
X \circ Y=\sum_{k=0}^{\infty} \frac{1}{k !}(X, Y, \underbrace{x, \ldots, x}_{k \text { times }}),
$$

where $x=x^{a} e_{a}$, and $\circ$ is the product on $H_{K}$ defined by (4).

Proof. Notice that each $Y_{n}$ is symmetric, then we have

$$
\begin{aligned}
g\left(e_{a} \circ e_{b}, e_{c}\right) & =\frac{\partial^{3} \Phi}{\partial x^{a} \partial x^{b} \partial x^{c}}=\sum_{n \geq 3} \frac{1}{n !} \frac{\partial^{3}}{\partial x^{a} \partial x^{b} \partial x^{c}} Y_{n}(\underbrace{x, \ldots, x}_{n \text { times }}) \\
& =\sum_{n \geq 3} \frac{n(n-1)(n-2)}{n !} Y_{n}(e_{a}, e_{b}, e_{c}, \underbrace{x, \ldots, x}_{n-3 \text { times }}) \\
& =g\left(\sum_{k=0}^{\infty} \frac{1}{k !}(e_{a}, e_{b}, \underbrace{x, \ldots, x}_{k \text { times }}), e_{c}\right) .
\end{aligned}
$$

From Lemma 2.1, we see that a formal Frobenius manifold structure has an identity if and only if we have

$$
\left(1, x_{1}, \ldots, x_{n}\right)= \begin{cases}x_{1}, & \text { for } n=1 \\ 0, & \text { otherwise }\end{cases}
$$

\section{Tautological constructions}

Theorem 3.1. If $(H, \cdot, g)$ is a Frobenius algebra, then

$$
\Phi(x)=\sum_{n \geq 3} \frac{1}{n !} g\left(x^{n-1}, x\right),
$$

is a formal Frobenius manifold structure with $(H, \cdot, g)$ as initial data. 
Proof. Clearly we have

$$
\begin{aligned}
& Y_{n+1}\left(x_{1}, \ldots, x_{n}, x_{n+1}\right)=g\left(x_{1} \cdots x_{n}, x_{n+1}\right), \\
& \left(x_{1}, \ldots, x_{n}\right)=x_{1} \cdots x_{n} .
\end{aligned}
$$

Since $(H, \cdot, g)$ is a Frobenius algebra, (6) and (7) are easily verified.

It is clear that the normalized potential function $\widetilde{\Phi}(x)$ is

$$
\widetilde{\Phi}(x)=\sum_{n=1}^{\infty} \frac{1}{n !} \int x^{n}=\int\left(e^{x}-1\right)=\int e^{x} .
$$

Here we assume that $\int 1=0$. Compare with (3.18) in Dijkgraaf-VerlindeVerlinde [9] and (3.17) in Witten [21].

Now we assume that the Frobenius algebra $(H, \cdot g)$ has an identity 1, equivalently, there is an injective $\mathbf{k}$-algebra homomorphism $\epsilon: \mathbf{k} \rightarrow H$. Assume that $H$ has an augmentation, i.e., a k-algebra homomorphism $\mu: H \rightarrow \mathbf{k}$ such that $\mu \epsilon=i d_{\mathbf{k}}$. Let $\bar{H}=\mu^{-1}(0)$ be the augmentation ideal. Then we have a decomposition

$$
H=\epsilon(\mathbf{k}) \oplus \bar{H} .
$$

For $x \in H$, set $\bar{x}=x-\epsilon \mu(x)$. If $x \in \bar{H}$, then $\bar{x}=x$.

Theorem 3.2. If $(H, \cdot)$ is an augmented commutative associative algebra with identity, then

$$
\begin{aligned}
\left(x_{1}, x_{2}\right) & =x_{1} x_{2}, \\
\left(x_{1}, \ldots, x_{n}\right) & =\bar{x}_{1} \cdots \bar{x}_{n}, \quad n>2,
\end{aligned}
$$

defines on $H$ a Comm $_{\infty}$-algebra structure which satisfies (9).

Proof. Since all the $x_{s_{j}}$ 's in (6) appear as $\bar{x}_{s_{j}}$, if any $x_{s_{j}}$ lies in $\epsilon(\mathbf{k})$, then both sides of (6) vanish. So we can assume that all the $x_{s_{j}}$ 's lie in $\bar{H}$. If $a=1$, then the only possibly nonzero term on the left hand side of (6) is

$$
\left((1, b), c, x_{1}, \ldots, x_{n}\right)=\left(b, c, x_{1}, \ldots, x_{n}\right),
$$

while the only possibly nonzero term on the right hand side is

$$
\left(1,\left(b, c, x_{1}, \ldots, x_{n}\right)\right)=\left(b, c, x_{1}, \ldots, x_{n}\right) .
$$

Then (6) holds. So we can assume that $a \in \bar{H}$. We can similarly treat the case of $b=1$ or $c=1$. Now if $a, b, c$ and all $x_{s_{j}}$ lie in $\bar{H}$, then clearly (6) holds.

Let $(H, \cdot g)$ be an augmented Frobenius algebra with identity. If we set

$$
Y_{n+1}\left(x_{1}, \ldots, x_{n+1}\right)=g\left(\bar{x}_{1} \cdots \bar{x}_{n}, x_{n+1}\right),
$$

then it is easy to see that $Y_{n+1}$ is not cyclic. We shall instead set

$$
Y_{n+1}\left(x_{1}, \ldots, x_{n+1}\right)=g\left(\bar{x}_{1} \cdots \bar{x}_{n}, \bar{x}_{n+1}\right) .
$$

To find the higher multiplication $\left(x_{1}, \ldots, x_{n}\right)$ this defines, notice that

$$
g\left(\bar{x}_{1} \cdots \bar{x}_{n}, \bar{x}_{n+1}\right)=g\left(\bar{x}_{1} \cdots \bar{x}_{n}, x_{n+1}-\epsilon \mu\left(x_{n+1}\right)\right) .
$$


We need to find a linear operator $p$ such that

$$
g\left(\bar{x}_{1} \cdots \bar{x}_{n}, \epsilon \mu\left(x_{n+1}\right)\right)=g\left(p\left(\bar{x}_{1} \cdots \bar{x}_{n}\right), x_{n+1}\right) .
$$

To this end, we assume that there is an element $v \in \bar{H}$ and a decomposition

$$
\bar{H}=\mathbf{k} v \oplus \widetilde{H},
$$

such that $g(1, v)=1, g(x, 1)=g(x, v)=0$ for $x \in \widetilde{H}$. We also assume $g(1,1)=$ $g(v, v)=0$. Since $\int v=1, v$ is called the volume element of $(H, \cdot, g)$. Let $p: H \rightarrow \mathbf{k} v$ be the projection given by $x \mapsto\left(\int x\right) v$. Then we have

$$
\begin{aligned}
g\left(\bar{x}_{1} \cdots \bar{x}_{n}, \epsilon \mu\left(x_{n+1}\right)\right)= & g\left(p\left(\bar{x}_{1} \cdots \bar{x}_{n}\right), \epsilon \mu\left(x_{n+1}\right)\right)= \\
& g\left(p\left(\bar{x}_{1} \cdots \bar{x}_{n}\right), x_{n+1}-\bar{x}_{n+1}\right)=g\left(p\left(\bar{x}_{1} \cdots \bar{x}_{n}\right), x_{n+1}\right) .
\end{aligned}
$$

Therefore, $\left(x_{1}, \ldots, x_{n}\right)=\bar{x}_{1} \cdots \bar{x}_{n}-p\left(\bar{x}_{1} \cdots \bar{x}_{n}\right)$.

Theorem 3.3. Suppose $(H, \cdot, g)$ is an augmented Frobenius algebra with identity and a volume element $v$, such that $x \cdot v=0$ for any $x \in \bar{H}$. Then

$$
\bar{\Phi}(x)=\frac{1}{3 !} g\left(x^{2}, x\right)+\sum_{n>3} \frac{1}{n !} g\left(\bar{x}^{n-1}, \bar{x}\right)=\frac{1}{3 !} \int x^{3}+\sum_{n>3} \frac{1}{n !} \int \bar{x}^{n},
$$

is a formal Frobenius manifold structure with $(H, \cdot, g)$ as initial data.

Proof. We have

$$
\begin{aligned}
Y_{3}\left(x_{1}, x_{2}, x_{3}\right) & =g\left(x_{1} x_{2}, x_{3}\right), \\
\left(x_{1}, x_{2}\right) & =x_{1} x_{2},
\end{aligned}
$$

and for $n>2$,

$$
\begin{aligned}
Y_{n+1}\left(x_{1}, \ldots, x_{n+1}\right) & =g\left(\bar{x}_{1} \cdots \bar{x}_{n}, \bar{x}_{n+1}\right), \\
\left(x_{1}, \ldots, x_{n}\right) & =\bar{x}_{1} \cdots \bar{x}_{n}-p\left(\bar{x}_{1} \cdots \bar{x}_{n}\right) .
\end{aligned}
$$

Then (7) and (9) are clearly satisfied. We now verify (6). As in the proof of Theorem 3.2, we can assume that $a, b, c$ and all $x_{s_{j}}$ 's lie in $\bar{H}$. Now since the multiplication of $v$ with any element in $\bar{H}$ vanishes, we have

$$
\begin{aligned}
\sum_{S_{1} \amalg S_{2}}\left(\left(a, b, x_{S_{1}}\right), c, x_{S_{2}}\right) & =\sum_{S_{1} \amalg S_{2}}\left(a b x_{S_{1}}-\left(\int a b x_{S_{1}}\right) v, c, x_{S_{2}}\right) \\
& =\sum_{S_{1} \amalg S_{2}} a b x_{S_{1}} c x_{S_{2}}-\sum_{S_{1} \amalg S_{2}}\left(\int a b x_{S_{1}} c x_{S_{2}}\right) v \\
& =2^{n}\left(a b c x_{1} \cdots x_{n}-p\left(a b c x_{1} \cdots x_{n}\right)\right) .
\end{aligned}
$$

Similarly, the calculation for

$$
\sum_{S_{1} \amalg S_{2}}\left(a,\left(b, c, x_{S_{1}}\right), x_{S_{2}}\right),
$$

yields the same result. The proof is complete. 
Remark 3.1. Write $x=x^{a} e_{a}=x^{0} 1+\bar{x}$. Then the normalized potential function is given by

$$
\widetilde{\bar{\Phi}}(x)=\frac{x_{0}^{2}}{2} \int \bar{x}+\frac{x_{0}}{2} \int \bar{x}^{2}+\sum_{n \geq 1} \frac{1}{n !} \int \bar{x}^{n} .
$$

When $\mathbf{k}=\mathbb{R}$ or $\mathbb{C}, H$ is isomorphic to $\mathbb{R}^{n}$ or $\mathbb{C}^{n}$, and so it has an induced metric from the standard metric on $\mathbb{R}^{n}$ or $\mathbb{C}^{n}$. Then there are constants $c_{1}$ and $c_{2}$ such that

$$
\left|\int x\right| \leq c_{1}|x|, \quad|x \cdot y| \leq c_{2}|x||y|,
$$

Note also $|\bar{x}| \leq|x|$. Then we have

$$
\left|\frac{1}{n !} \int x^{n}\right| \leq \frac{c_{1} c_{2}^{n}|x|^{n}}{n !}, \quad\left|\frac{1}{n !} \int \bar{x}^{n}\right| \leq \frac{c_{1} c_{2}^{n}|x|^{n}}{n !} .
$$

Therefore, the formal power series $\Phi$ in Theorem 3.1 and $\Phi$ in Theorem 3.3 both converge for all $x \in H$. Hence we obtain self-parameterizing family of Frobenius algebras from our constructions.

Remark 3.2. Since Theorem 3.1 applies to any Frobenius algebra $H$, we can apply it to $H_{K}$ with deformed product $\circ$. Hence we get family of families. Similarly, if $H$ is a Frobenius algebra with identity which satisfies the conditions in Theorem 3.3, then we get a Frobenius algebra $\left(H_{K}, \bar{o}, g\right)$ which still satisfies those conditions with the naturally extended augmentation and the same volume element. So we can apply Theorem 3.3 to it to get a family of families. This procedure can certainly be iterated for as many times as we wish.

\section{Tautological Frobenius structure on de Rham cohomology}

There are easy generalizations of the above results to graded situations. For graded version of WDVV equations and formal Frobenius manifold structures, the interested reader can consult Manin [17]. The examples of graded Frobenius algebra we are particularly interested in are the de Rham cohomology of a closed oriented smooth manifolds, the Dolbeault cohomology ring of a closed complex manifolds, and $H^{-*, *}(M)$ of a closed Calabi-Yau manifold. When the manifold is connected, these Frobenius algebras have identities. Clearly, they have volume forms satisfying the conditions in Theorem 3.3, so we get tautological constructions of formal Frobenius manifold structures with identities on them. In the case of de Rham cohomology, we call the result tautological Frobenius structure on the de Rham cohomology.

We now compute the potential function for projective spaces. Let $\omega \in$ $H^{2}\left(\mathbb{C P}_{n}\right)$ be a generator of the cohomology ring $H^{*}\left(\mathbb{C P}_{n}\right)$. We assume that $\int_{\mathbb{C P}_{n}} \omega^{n}=1$. Take $e_{j}=\omega^{j}$, and denote the coordinates in $\left\{1, \omega, \ldots, \omega^{n}\right\}$ by 
$x_{0}, \ldots, x_{n}$. Then

$$
\begin{aligned}
\widetilde{\bar{\Phi}}_{\mathbb{C P}_{n}}\left(x_{0}, \ldots, x_{n}\right)= & \frac{1}{2} x_{0}^{2} x_{n} \\
+ & \frac{1}{2} x_{0} \int_{\mathbb{C P}_{n}}\left(x_{1} \omega+\cdots x_{n} \omega^{n}\right)^{2} \\
& +\sum_{m \geq 1} \frac{1}{m !} \int_{\mathbb{C P}_{n}}\left(x_{1} \omega+\cdots x_{n} \omega^{n}\right)^{m} \\
= & \frac{1}{2} x_{0}^{2} x_{n}++\frac{1}{2} x^{0} \sum_{\substack{i+j=n \\
1 \leq i, j \leq n}} x_{i} x_{n-i} \\
& +\sum_{m \geq 1} \frac{1}{m !} \sum_{\substack{i_{1} j_{1}+\cdots i_{m} j_{m}=n \\
1 \leq i_{l} \leq n, 0 \leq j_{l} \leq m}} x_{i_{1}}^{j_{1}} \cdots x_{i_{m}}^{j_{m}} .
\end{aligned}
$$

Since this is a polynomial, it is of course different from the potential function for quantum cohomology of $\mathbb{C P}_{n}$ which contains $e^{x_{1}}$ (see e.g., Kontsevich-Manin [14], §5). The generating functional for these potential functions has a very simple form. We set $\bar{x}(q)=x_{1} q+x_{2} q^{2}+\cdots+x_{n} q^{n}+\cdots$. For a polynomial $\phi$ in $q$, denote by $\phi_{(n)}$ the degree $n$ part of $\phi$. Then we have

$$
\begin{aligned}
& \sum_{n \geq 1} \sum_{\substack{i+j=n \\
1 \leq i, j \leq n}} x_{i} x_{n-i} q^{n}=\sum_{n \geq 1} \sum_{\substack{i+j=n \\
1 \leq i, j \leq n}}\left(x_{i} q^{i}\right)\left(x_{j} q^{j}\right) \\
&=\sum_{n \geq 1}\left[\left(x_{1} q+\cdots+x_{n} q^{n}\right)^{2}\right]_{(n)} \\
&=\sum_{n \geq 1}\left(x_{1} q+\cdots+x_{n} q^{n}+\cdots\right)_{(n)}^{2}=\bar{x}(q)^{2} \\
& \sum_{n \geq 1} \sum_{m \geq 1} \frac{1}{m !} \sum_{\substack{i_{1} j_{1}+\cdots i_{m} j_{m}=n \\
1 \leq i_{l} \leq n, 0 \leq j_{l} \leq m}} x_{i_{1}}^{j_{1}} \cdots x_{i_{m}}^{j_{m}} q^{n} \\
&=\sum_{n \geq 1} \sum_{m \geq 1} \frac{1}{m !} \sum_{\substack{i_{1} j_{1}+\cdots i_{m} j_{m}=n \\
1 \leq i_{l} \leq n, 0 \leq j_{i} \leq m}}\left(x_{i_{1}} q^{i_{1}}\right)^{j_{1}} \cdots\left(x_{i_{m}} q^{i_{m}}\right)^{j_{m}} \\
&=\sum_{n \geq 1} \sum_{m \geq 1} \frac{1}{m !}\left[\left(x_{1} q+\cdots+x_{n} q^{n}\right)^{m}\right]_{(n)} \\
&=\sum_{n \geq 1} \sum_{m \geq 1} \frac{1}{m !}\left[\left(x_{1} q+\cdots+x_{n} q^{n}+\cdots\right)^{m}\right]_{(n)} \\
&=\sum_{m \geq 1} \frac{1}{m !}\left(x_{1} q+\cdots+x_{n} q^{n}+\cdots\right)^{m}=e^{\bar{x}(q)}-1 .
\end{aligned}
$$


Therefore,

$$
\begin{aligned}
\overline{\mathcal{F}}(q, x) & :=1+\sum_{n \geq 1} \widetilde{\bar{\Phi}}_{\mathbb{C P}_{n}}\left(x_{0}, \ldots, x_{n}\right) q^{n} \\
& =\frac{1}{2} x_{0}^{2} \bar{x}(q)+\frac{1}{2} x_{0} \bar{x}(q)^{2}+e^{\bar{x}(q)}
\end{aligned}
$$

Similarly,

$$
\begin{aligned}
\widetilde{\Phi}_{\mathbb{C P}_{n}}\left(x_{0}, \ldots, x_{n}\right) & =\sum_{m \geq 1} \frac{1}{m !} \int_{\mathbb{C P}_{n}}\left(x_{0} 1+x_{1} \omega+\cdots x_{n} \omega^{n}\right)^{m} \\
& =\sum_{m \geq 1} \sum_{k=0}^{m} \frac{x_{0}^{m-k}}{(m-k) !} \frac{1}{k !} \int_{\mathbb{C P}_{n}}\left(x_{1} \omega+\cdots x_{n} \omega^{n}\right)^{k} \\
& =\sum_{m \geq 1} \sum_{k=1}^{m} \frac{x_{0}^{m-k}}{(m-k) !} \frac{1}{k !}\left[\left(x_{1} q+\cdots+x_{n} q^{n}\right)^{k}\right]_{(n)} / q^{n} \\
& =\sum_{l \geq 0} \frac{1}{l !} x_{0}^{l} \sum_{k \geq 1} \frac{1}{k !}\left[\left(x_{1} q+\cdots+x_{n} q^{n}+\cdots\right)^{k}\right]_{(n)} / q^{n} \\
& =e^{x_{0}} \sum_{k \geq 1}\left[\bar{x}(q)^{k}\right]_{(n)} / q^{n} .
\end{aligned}
$$

then we have

$$
\begin{aligned}
\mathcal{F}(q, x) & :=1+\sum_{n \geq 1} \widetilde{\bar{\Phi}}_{\mathbb{C P}_{n}}\left(x_{0}, \ldots, x_{n}\right) q^{n} \\
& =1+\sum_{n \geq 1} e^{x_{0}} \sum_{k \geq 1} \frac{1}{k !}\left[\bar{x}(q)^{k}\right]_{(n)}=1+e^{x_{0}} \sum_{k \geq 1} \frac{1}{k !} \bar{x}(q)^{k} \\
& =1+e^{x_{0}}\left(e^{\bar{x}(q)}-1\right)=1-e^{x_{0}}+e^{x(q)}
\end{aligned}
$$

where $x(q)=x_{0}+\bar{x}(q)=x_{0}+x_{1} q+\cdots+x_{n} q^{n}+\cdots$. The computations for $\mathbb{H}_{n}$ are exactly the same. For a reader who is familiar with the Schur polynomials, the above calculation can be slightly simplified. Recall that such polynomials are defined by

$$
e^{\sum_{j \geq 1} x_{j} q^{j}}=\sum_{n \geq 0} p_{n}\left(x_{1}, \ldots, x_{n}\right) q^{n}
$$

Hence

$$
\sum_{m \geq 1} \frac{1}{m !} \sum_{\substack{i_{1} j_{1}+\cdots i_{m} j_{m}=n \\ 1 \leq i_{l} \leq n, 0 \leq j_{l} \leq m}} x_{i_{1}}^{j_{1}} \cdots x_{i_{m}}^{j_{m}}=p_{n}\left(x_{1}, \ldots, x_{n}\right) .
$$

\section{References}

[1] S. Barannikov and M. Kontsevich, Frobenius manifolds and formality of lie algebras of polyvector fields, Internat. Math. Res. Notices (1998), 201-215, alg-geom/9710032.

[2] H-D. Cao and J. Zhou, On quantum de Rham cohomology theory, Electron. Res. Announc. Amer. Math. Soc. 5 (1999), 24-34, math.DG/9804145.

[3] _ On quantum de Rham cohomology, math.DG/9806157. 
[4] - Frobenius manifold structure on Dolbeault cohomology and mirror symmetry, to appear in Comm. Anal. Geom., math.DG/9805094.

[5] __, Identification of two Frobenius manifolds, Math. Res. Lett. 6 (1999), 17-29, math.DG/9805095.

[6] , DGBV algebras and Frobenius manifolds from hyperkähler manifolds, TAMU preprint.

[7] _ Formal Frobenius manifold structure on equivariant cohomology, Commun. Contemp. Math. 1 (1999), 535-552 math.DG/9903024.

[8] K.-T. Chen, Extension of $C^{\infty}$ function algebra by integrals and Malcev completion of $\pi_{1}$, Advances in Math. 23 (1977), 181-210.

[9] R. Dijkgraaf, H. Verlinde, and E. Verlinde, Topological strings in $d<1$, Nuclear Phys. B 352 (1991), 59-86.

[10] B. Dubrovin, Geometry and analytic theory of Frobenius manifolds, Proceedings of the International Congress of Mathematicians, Vol. II (Berlin, 1998), Doc. Math. Extra Vol. II (1998), 315-326 (electronic), math. AG/9807034.

[11] M. Gerstenhaber, On the deformation of rings and algebras, Ann. of Math. (2) 79 (1964), 59-103.

[12] E. Getzler, Operads and moduli spaces of genus 0 Riemann surfaces, The moduli space of curves (Texel Island, 1994), 199-230, Progr. Math., 129, Birkhäuser Boston, Boston, MA, 1995, alg-geom/9411004.

[13] V.K.A.M. Gugenheim and J.D. Stasheff, On perturbations and $A_{\infty}$-structures, Bull. Soc. Math. Belg. Sér. A 38 (1986), 237-246 (1987).

[14] M. Kontsevich and Yu. Manin, Gromov-Witten classes, quantum cohomology, and enumerative geometry, Comm. Math. Phys. 164 (1994), 525-562, hep-th/9402147.

[15] P.S. Landweber (Ed.), Elliptic curves and modular forms in algebraic topology, Proceedings of a conference held in Princeton, New Jersey, September 15-17, 1986, Lecture Notes in Mathematics, 1326. Springer-Verlag, Berlin-New York, 1988.

[16] Yu. Manin, Frobenius manifolds, quantum cohomology, and moduli spaces. American Mathematical Society Colloquium Publications, 47. American Mathematical Society, Providence, RI, 1999. (Preprint MPI 96-113).

[17] Y. Manin, Three constructions of Frobenius manifolds: a comparative study, Asian J. Math. 3 (1999), 179-220, math.QA/9801006.

[18] S.A. Merkulov, Formality of canonical symplectic complexes and Frobenius manifolds, Internat. Math. Res. Notices (1998), 727-733, math.SG/9805072.

[19] _ Strong homotopy algebras of a Kähler manifold, Internat. Math. Res. Notices (1999), 153-164, math.AG/9809172.

[20] Y. Ruan and G. Tian, A mathematical theory of quantum cohomology, J. Differential Geom. 42 (1995), 259-367.

[21] E. Witten, Two-dimensional gravity and intersection theory on moduli space, Surveys in differential geometry (Cambridge, MA, 1990), 243-310, Lehigh Univ., Bethlehem, PA, 1991.

[22] J. Zhou, Hodge theory and $A_{\infty}$ structures on cohomology, to appear in Intern. Math. Res. Notices, Math.DG/9903154.

[23] - Homological perturbation theory and mirror symmetry, math.DG/9906096.

Department of Mathematics, Texas A\&M University, College Station, TX 77843

E-mail address: zhou@math.tamu.edu 